\title{
Acute appendicitis in elderly during Covid-19 pandemic
}

\author{
G. Lisi ${ }^{1}$ - M. Campanelli ${ }^{2}$ M. R. Mastrangeli ${ }^{1} \cdot$ S. Grande ${ }^{2} \cdot$ M. A. Viarengo ${ }^{3} \cdot$ G. M. Garbarino ${ }^{4} \cdot$ G. Vanni $^{2}$. \\ M. Grande ${ }^{2}$
}

Accepted: 21 May 2021 / Published online: 27 May 2021

(c) The Author(s), under exclusive licence to Springer-Verlag GmbH Germany, part of Springer Nature 2021

\begin{abstract}
Purpose During the past months, the Italian Government has reduced the restrictions and access to hospitals. Since then, up to $40 \%$ of non-traumatic abdominal emergencies have had unusual delayed treatment. Given the rapidly evolving situation and the absence of evidence to support recommendations during the pandemic, it is useful to assess how the current situation is influencing the management of elderly with acute appendicitis.

Methods Between February 2020 and December 2020, all patients older than 68 years old undergone appendectomy were included. Surgical approach, hospital stay, post-operative complications, radiology reports, and histologic examination were included in the retrospective analysis and compared with a same sample of the same period before the pandemic.

Results Twenty-seven patients underwent appendectomy for acute appendicitis during the pandemic and 34 patients in the pre-outbreak period. Laparoscopic approach was completed in $51.8 \%$ of the cases, while conversion to laparotomy in $22.3 \%$ of patients and open procedure in the $25.9 \%$, before the pandemic $73.6 \%, 14.7 \%$, and $11.7 \%$, respectively. During the pandemic, complicated appendicitis occurred in $59.3 \%$ of the cases (26.5\% before the outbreak). One patient was treated for a pelvic abscess, while no minor complications were detected. No mortality rate was reported, with a mean hospital stay of 5.64 days during the outbreak and 4.21 days before the pandemic.

Conclusion Our data highlighted a partial delay in diagnosis in the elderly group, and an increase in complicated appendicitis also demonstrated by the need for conversion to laparotomy.
\end{abstract}

Keywords Covid-19 · Acute appendicitis · Appendectomy $\cdot$ Elderly $\cdot$ Laparoscopy

\section{Introduction}

Acute appendicitis (AA) is a common cause of abdominal pain and one of the most frequent surgical conditions in emergency, which can turn into perforation and peritonitis. The male/female ratio is $1.4 / 1$ with a lifetime risk of $8.6 \%$ in males and $6.7 \%$ in females, while the risk of undergoing an appendectomy is much

G. Lisi

giolimas06@yahoo.it

1 Department of Surgery, Sant'Eugenio Hospital, Piazzale dell'Umanesimo 10, 00144 Rome, Italy

2 Emergency Surgery Unit, University Hospital of Tor Vergata, viale Oxford 81, 00133 Rome, Italy

3 Day-Case Surgery Unit, Israelitic Hospital, 00144 Rome, Italy

4 Department of Medical Surgical Sciences and Translational Medicine, Sant'Andrea University, Via di Grottarossa, 1035-39, 00189 Rome, Italy lower in men than in women ( $12 \%$ vs. $23 \%$, respectively) [1, 2]. Nonetheless, during the last decades, there was a progressive growth of appendicitis incidence among elderly, probably related to the increase of mean life expectancy and improvement of diagnosis and imaging techniques $[3,4]$.

Perforated appendicitis represents $20 \%$ of all appendicitis and significantly increases the rate of post-operative events. The rate of complicated appendicitis is higher in elderly patients than in young: perforation rates reach $70 \%$ and morbidity up to $48 \%$ [4].

During the last year, the University Hospital of "Tor Vergata" Rome (Italy) was named "Covid Hospital-4," the fourth referral center in Rome for COVID-19-positive patients. Over the past months, the Italian Government has reduced the restrictions and access to hospitals as well as for outpatient care. Since then, up to $40 \%$ of non-traumatic abdominal emergencies have had unusual delayed treatment $[5,6]$. In our country, about $40 \%$ of surgeons reported an unusual delay in the presentation of non-traumatic 
abdominal emergencies. In all cases, the fever had been present for several days before hospitalization. The delay was partially related to patient choice, preferring to stay at home until symptoms worsened, and in part due to the waiting list for the COVID-19 emergency room test, almost half of the centers reported emergency associated with a more serious presentation due to a diagnostic delay [7]. The authorities have asked many patients with fever not to go to the hospital if they do not have breathing difficulties. In these patients, fever may not be caused by COVID-19-related pneumonia but by an abdominal infection. There are also numerous patients whose diagnostic delay is related to the time spent in the emergency room, because of the scarcity of hospital beds available and because of the diagnostic work for COVID-19.

Given the rapidly evolving situation and the absence of evidence to support recommendations during the COVID-19 pandemic, it is useful to assess how the current situation affected the management of elderly patients with acute appendicitis. The addressed questions were as follows: whether there was a delay in the presentation and diagnosis of acute appendicitis in elderly, how age-related diversities could influence clinical onset and consider the best diagnostic tool, and surgical strategy also comparing these data with the sample in a pre-pandemic period.

\section{Methods and results}

We retrospective analyzed all patients older than 68 years old who underwent appendectomy at the Emergency Surgery Unit of Tor Vergata University, between February 2020 and December 2020, comparing them with the same sample from February 2019 to December 2019. Patient demographics, COVID status with pre-operative rapid antigen test, preand post-operative management, surgical approach, length of stay, and 30-day complications were recorded. We excluded all patients that underwent appendectomy for malignant disease or received appendectomy during elective surgery, and patients with negative acute appendicitis (AA) at the histologic examination. According to the recent study of Kurian et al. [8], 68 years old seems to be the ideal cut-off dividing young from old patients who undergo gastrointestinal procedures. The items considered in the analysis were age, sex, temperature, and white blood cell (WBC) at admission and radiology reports. Other items included were surgical approach (laparoscopy and laparotomy), histologic examination, time of hospital stay, and post-operative complications.

As shown in Table 1 is comparison of the two groups of elderly analyzed in this report. During the pandemic, 27 patients with a mean age of 74.8 years old (range 68 to 82 years) were included, while before the outbreak, 34 patients with a mean age of 76.4 years old (range 68 to 79 years) were enrolled. Of the 27 patients tested for Covid-19 on admission, none of the patients were positive. WBC at the moment of admission was $14.5 \pm 7.6$ (mila/ug), while $\mathrm{C}$ reactive protein $(\mathrm{CRP})$ was $163.08 \pm 103.81(\mathrm{mg} / \mathrm{l})$, despite body temperature showed no significant variations (37.01 $\left.{ }^{\circ} \mathrm{C} \pm 0.66\right)$. Every patient received the same antimicrobial therapy, III generation cephalosporine, and metronidazole, started from the moment of diagnosis until the discharge. CT scan was performed in all patients. Laparoscopic approach
Table 1 Pre- and post-operative data

\begin{tabular}{lll}
\hline & Pts during CoV-19 (27) & Pts pre-CoV-19 (34) \\
\hline Clinical exams & & \\
WBC (mila/ug) & $14.5 \pm 7.6$ & $12.4 \pm 5.6$ \\
CRP (mg/L) & $163.08 \pm 103.81$ & $78.2 \pm 45.63$ \\
Temperature $\left({ }^{\circ} \mathrm{C}\right)$ & $37.01 \pm 0.66$ & $37 \pm 0.95$ \\
Pre-operative Imaging & & \\
US (\%) & 0 & 0 \\
CT (\%) & $27(100)$ & $34(100)$ \\
Surgical approach & & $25(73.6)$ \\
Laparoscopy (\%) & $14(51.8)$ & $4(11.7)$ \\
Open (\%) & $7(25.9)$ & $5(14.7)$ \\
Conversion to open (\%) & $6(22.3)$ & $9(26.5)$ \\
Histologic examination & & $16(47)$ \\
Appendicitis (\%) & $4(14.8)$ & $9(26.5)$ \\
Acute appendicitis (\%) & $7(25.9)$ & \\
Complicated appendicitis (\%) & $16(59.3)$ & 0 \\
Post-operative data & $1(3.7)$ & $4(11.7)$ \\
Major complications (\%) & 0 & 0 \\
Minor complications (\%) & 0 & $4.21 \pm 2.01$ \\
Mortality (\%) & $5.64 \pm 2.67$ & \\
Mean hospital stay (days) & &
\end{tabular}

Pts patient, Yrs years, CRP C reactive protein 
was completed in $51.8 \%$ of the cases, while conversion to laparotomy was necessary in the $22.3 \%$ of patients and open procedure was performed in 7 of 27 (25.9\%) patients. Complicated appendicitis (CA) was occurred in 16 of the 27 patients (59.3\%) despite no significant relationship between these findings and the histologic examination was reported; conversely, CA was lower before the outbreak $(26.5 \%, 9$ patients). This data could confirm the hypothesis of a diagnostic delay due to the pandemic. Among post-operative complications, there was one patient treated for a pelvic abscess while no minor complications were detected during the pandemic. Moreover, no mortality rate was reported in both groups; moreover, the mean hospital stay was 5.64 days (range 3 to 8 days) during the pandemic and 4.21 days (range 2 to 6 days) before the outbreak.

\section{Considerations and conclusions}

In Italy, we are taking care of increasingly elderly patients in elective as well as emergency surgery. According to our data compared with the literature [9], there was no difference among admission parameters, even if clinical onset in elderly patients was often localized throughout the abdomen; moreover, no differences in terms of WBC (mila/ug), CRP $(\mathrm{mg} / \mathrm{l})$, and body temperature were detected.

Segev et al. [10] hypothesized the use of the CT scan in the elderly population is due to the clinical onset, often more complicated with previous gastrointestinal surgery or disease. According to our pre-Covid (CoV)-19 and during CoV-19 sample size, we could confirm that CT scan is the gold standard diagnostic tool for acute appendicitis in elderly patients possibly due to the high risk of abdominal malignancies; other diagnostic exams may not be helpful, and they might delay the time of diagnosis with the risk of a worse clinical scenario [11]. Di Saverio et al. [9] in their recent analysis have demonstrated that the volume of radiological examinations for acute appendicitis decreased overall and the proportion of severe cases increased during the pandemic period. The results of their study showed a reduction of $61 \%$ in the total number of abdominal CT scans performed in patients with signs of appendicitis. However, these appendicities diagnosed by $\mathrm{CT}$ are presenting at later stages with increased disease severity; the results seem congruent with the initial hypothesis that people are postponing visiting the emergency room until their symptoms become severe during the acute pandemic period.

Contrary to the few study published, Turanli and Kiziltan [12] analyzed two hundred and fourteen patients before and during the pandemic. Although there was a slight increase in perforation during the pandemic period, this negativity was not statistically significant $(p=0.58)$; he concluded there were no difference in the patients before and during the outbreak in the rate of perforated appendicitis in patients who were referred from other pandemic hospitals (29.4\%) and those admitted via their own emergency room (16.6\%) $(p=0.27)$ during the pandemic period.

These data referred to a specific sample size with a median age of 39 years old; it may be a confounder factor and may reduce the power of the analysis as well as underrate the complications associated with acute appendicitis in elderly.

According to the literature [13], complicated appendicitis is mostly reported in elderly patients (39.2\%) than younger (10.5\%) not it was occurred in $26.5 \%$ ( 9 of 34 patients) prepandemic and 59.3\% (16 of 27 patients) during the outbreak.

Most of the patient (51.8\% vs $73.6 \%$ ) underwent laparoscopic procedure in both groups, and this data may demonstrate that the laparoscopic approach represents the most frequent used treatment, independent of age despite the pandemic and the possible diagnostic delay.

In addition, Zhou and Cen [14] in his recent retrospective analysis of 67 patients with a mean age of 40.9 years old reported the ratio of open appendectomies was higher than in a pre-pandemic period, and therefore, the amount of blood loss increased due to the surgical method. Furthermore, the higher proportion of perforated appendicitis was also an important cause of increased blood loss.

During the pandemic, conversion to laparotomy occurred in six patients ( $22.3 \%$ vs $14.7 \%$ ), confirming what is reported in literature. Moreover, in six patients, laparoscopy was converted to laparotomy because of a complicated scenario, confirmed by histologic examination (abscess and acute appendicitis with wide wall necrosis) [12].

Recently, Javanmard-Emamghissi et al. [15] in his UK cohort study analyzed 500 patients from 48 sites affected by acute appendicitis and treated conservatively or surgically; nevertheless, a previous survey in Ireland [13] showed that $54 \%$ of acute appendicitis patients in the conservative care group had on-going discomfort on follow-up post-discharge. Sixty-three percent would choose up-front appendicectomy if they could decide again. Despite the author included all patients under 50 years old, he reported that two hundred and seventy-one (54\%) patients were initially treated conservatively, with only $26(10 \%)$ cases progressing to an operation. Operative interventions were performed laparoscopically in 44\% (93/211). At 30 days, complications were significantly higher in the operative group $(p<0.001)$; this finding might be due to an increase of laparotomy that it was not reported in our $<68$-year-old group. Within this study cohort, 56\% (133/237) patients having an operation had an open procedure. This is at significant odds with UK practice prior to the pandemic, where only a small number of patients were having open procedures $(0.4 \%)$ [16]. This is likely due to guidance issues that suggested laparoscopic surgery should be avoided due to concerns about aerosol generating 
procedures. These findings were also confirmed in the recent ACIE Appy Study where Ielpo B and coauthors highlighted that one-third of surgeons interviewed have changed their approach from laparoscopic to open surgery owing to the popular (but evidence-lacking) advice from expert groups during the initial phase of the pandemic although no agreement on how to filter surgical smoke plume during laparoscopy was identified [17].

The decision of proceeding straight to laparotomy was based on the clinical onset of the patient, the imaging, and the abdomen evaluation. This data may be partially related to the patient choice, preferring to stay at home until symptoms worsened, and in part due to the waiting list for the Covid-19 emergency room test, this choice was reported more frequent in elderly patients than the youngers $[15,17]$. Contrary to what reported in the literature [14, 15, 17], we carried no increase of morbidity. In fact, only one case required a percutaneous drainage due to a pelvic abscess.

Our report showed a small sample size, but certainly allows to look after appendectomy in elderly with positivism also during the pandemic. Our data highlighted a partial delay in diagnosis in the elderly group, and an increase in complicated appendicitis also demonstrated by the need for conversion to laparotomy despite no significant relationship between these findings and the histologic examination was reported.

Acknowledgements Lisi G design and wrote the paper; Campanelli M, Mastrangeli MR, and Grande S collected data; Viarengo MA and Garbarino GM made study design, Grande $M$ and Vanni G drafting the manuscript.

Availability of data and material Available.

\section{Declarations}

Conflict of interest The authors declare no competing interests.

\section{References}

1. Society of American Gastrointestinal and Endoscopic Surgeons (2020) SAGES and EAES recommendations regarding surgical response to COVID-19 crisis. https://www.sages.org/recommendations-surgicalresponse-covid-19/ [accessed 30 Mar 2020]

2. Di Saverio S, Khan M, Pata F, Ietto G, De Simone B, Zani E et al (2020) Laparoscopy at all costs? Not now during COVID19 and not for acute care surgery and emergency colorectal surgery. J Trauma Acute Care Surg 88:715-718

3. Royal College of Surgeons of England (2020) Updated intercollegiate general surgery guidance on COVID-19. https://www. rcseng.ac.uk/coronavirus/joint-guidance-for-surgeons-v2/ [accessed 7 Apr 2020]

4. American College of Surgeons (2020) COVID-19 guidelines for triage of emergency general surgery patients. https://www.facs.org/covid-19/ clinical-guidance/elective-case/emergency-surgery [accessed 15 Apr 2020]

5. An Y, Bellato V, Konishi T, Pellino G, Sensi B, Siragusa L et al (2020) Surgeons' fear of getting infected by COVID19: a global survey. Br J Surg 107:e543-e544

6. Lisi G, Campanelli M, Spoletini D, Carlini M (2020) The possible impact of COVID-19 on colorectal surgery in Italy. Colorectal Dis 22(6):641-642

7. Lisi G, Campanelli M, Villa M, Grande S, Grande M (2020) Colorectal emergency surgery in a COVID-19 referral hospital during the phase 2 in Italy. Int J Colorectal Dis 35(11):2137-2138

8. Kurian AA, Wang L, Grunkemeier G, Bhayani NH, Swanstrom LL (2013) Defining "the elderly" undergoing major gastrointestinal resections: receiver operating characteristic analysis of a large ACS-NSQIP cohort. Ann Surg 258(3):483-9

9. Di Saverio S, Podda M, De Simone B, Ceresoli M, Augustin G, Gori A, Boermeester M, Sartelli M, Coccolini F, Tarasconi A, De' Angelis N, Weber DG, Tolonen M, Birindelli A, Biffl W, Moore EE, Kelly M, Soreide K, Kashuk J, Ten Broek R, Gomes CA, Sugrue M, Davies RJ, Damaskos D, Leppäniemi A, Kirkpatrick A, Peitzman AB, Fraga GP, Maier RV, Coimbra R, Chiarugi M, Sganga G, Pisanu A, De' Angelis GL, Tan E, Van Goor H, Pata F, Di Carlo I, Chiara O, Litvin A, Campanile FC, Sakakushev B, Tomadze G, Demetrashvili Z, Latifi R, Abu-Zidan F, Romeo O, Segovia-Lohse H, Baiocchi G, Costa D, Rizoli S, Balogh ZJ, Bendinelli C, Scalea T, Ivatury R, Velmahos G, Andersson R, Kluger Y, Ansaloni L, Catena F (2020) Diagnosis and treatment of acute appendicitis: 2020 update of the WSES Jerusalem guidelines. World J Emerg Surg 15(1):27

10. Segev L, Keidar A, Schrier I, Rayman S, Wasserberg N, Sadot E (2015) Acute appendicitis in the elderly in the twenty-first century. J Gastrointest Surg 19(4):730-5

11. Farahbakhsh F, Torabi M, Mirzaee M (2020) A comparative study on the diagnostic validity of three scoring systems in the diagnosis of acute appendicitis in emergency centres. African J Emerg Med 10(3):132-135

12. Turanli S, Kiziltan G (2021) Did the COVID-19 Pandemic cause a delay in the diagnosis of acute appendicitis? World J Surg 45(1):18-22

13. Collard M, Lakkis Z, Loriau J, Mege D, Sabbagh C, Lefevre JH, Maggiori L (2020) Antibiotics alone as an alternative to appendectomy for uncomplicated acute appendicitis in adults: changes in treatment modalities related to the COVID-19 health crisis. J Visc Surg 157(3S1):S33-S42

14. Zhou Y, Cen LS (2020) Managing acute appendicitis during the COVID-19 pandemic in Jiaxing. China. World J Clin Cases 8(19):4349-4359

15. Javanmard-Emamghissi H, Boyd-Carson H, Hollyman M, Doleman B, Adiamah A, Lund JN, Clifford R, Dickerson L, Richards S, Pearce L, Cornish J, Hare S, Lockwood S, Moug SJ, Tierney GM (2020) COVID: HAREM (Had Appendicitis, Resolved/Recurred Emergency Morbidity/Mortality) Collaborators Group. The management of adult appendicitis during the COVID-19 pandemic: an interim analysis of a UK cohort study. Tech Coloproctol 1-11

16. RIFT study group (2019) Evaluation of appendicitis risk prediction models in adults with suspected appendicitis. Br J Surg 107:73-86

17. Ielpo B, Podda M, Pellino G, Pata F, Caruso R, Gravante G, Di Saverio S (2020) ACIE Appy Study Collaborative. Global attitudes in the management of acute appendicitis during COVID-19 pandemic: ACIE Appy Study. Br J Surg. https://doi.org/10.1002/ bjs.11999 